\title{
Estimated glomerular filtration rate, anemia and outcomes in patients with acute ischemic stroke
}

\author{
Huihui Liu', Yuesong Pan², Peng Wu², David Wang ${ }^{3}$, Huaguang Zheng, Hao Li, Yongjun \\ $\mathrm{Cao}^{1}$, Shoujiang You ${ }^{1}$, Yongjun Wang ${ }^{2}$, Chunfeng Liu'1, Yilong Wang². \\ ${ }^{1}$ The second affiliated hospital of Soochow University, Neurology, Suzhou, China. \\ 2Beijing Tiantan Hospital- Capital Medical University, Department of Neurology, Beijing, China. \\ 3 Illinois Neurological Institute Stroke Network- Sisters of the Third Order of St. Francis Healthcare System- \\ University of Illinois College of Medicine, Neurology, Peoria, USA.
}

Background and Aims: Anemia is highly prevalent in patients with low estimated glomerular filtration rate (eGFR), the combined effect of anemia and low eGFR on the prognosis of outcome after stroke has not been well studied.

Method: Data of ischemic stroke patients in the prospective cohort study of China National Stroke Registry was reviewed. According to the levels of eGFR and hemoglobin, patients were categorized into four groups: low eGFR $(<60 \mathrm{~mL} / \mathrm{min}$ per 1.73 $\mathrm{m}^{2}$ ) or normal eGFR ( $\geq 60 \mathrm{~mL} / \mathrm{min}$ per $\left.1.73 \mathrm{~m}^{2}\right)$ with or without anemia. Multivariable logistic regression method was used to analyze the association between low eGFR with or without anemia and the poor functional outcome, dependence, mortality and stroke reoccurrence at 3 months and 1 year after stroke.
Results: This study included 8,303 stroke patients. After adjustment for the confounding factors, low eGFR alone increased risk of mortality (Odds ratio $[O R]=1.50,95 \%$ confidence interval $[95 \% \mathrm{Cl}] 1.14-1.97)$, but anemia alone was not associated with any of poor outcome at 1-year of follow up. In patients with both low eGFR and anemia, there was an increased risk of poor functional outcome (OR 1.73, 95\% Cl 1.30-2.29), mortality (OR 2.64, 95\% Cl 1.94-3.59) and stroke reoccurrence (OR 1.42, 95\% Cl 1.06-1.91) during 1-year of follow up, except that the dependence (OR 1.17, 95\% Cl 0.83 1.64).

Table 1. Adjusted odds ratio of estimated glomerular filtration rate (eGFR) with anemia or not for poor outcome at 1 year

\begin{tabular}{|c|c|c|c|c|c|c|c|}
\hline Outcome & & $\mathbf{N}$ & $\mathrm{N}$ of Events (\%) & $\begin{array}{l}\text { Crude OR } \\
(95 \% \mathrm{Cl})\end{array}$ & $P$ value & $\begin{array}{c}\text { Adjusted OR } \\
(95 \% \mathrm{CJ})\end{array}$ & $P$ value \\
\hline \multirow{4}{*}{ Poor functional outcomet } & None of both & 1697 & 28.3 & 1 & - & 1 & - \\
\hline & Anemia with normal eGFR & 550 & 36.7 & $1.47(1.30-1.65)$ & $<0.001$ & $1.01(0.87-1.17)$ & 0.87 \\
\hline & Low eGFR without anemia & 259 & 52.6 & $2.82(2.34-3.39)$ & $<0.001$ & $1.07(0.84-1.37)$ & 0.57 \\
\hline & Anemia with low eGFR & 187 & 59.0 & $3.64(2.89-4.56)$ & $<0.001$ & $1.73(1.30-2.29)$ & 0.001 \\
\hline \multirow{4}{*}{ Dependenceł } & None of both & 1109 & 20.5 & 1 & - & 1 & - \\
\hline & Anemia with normal eGFR & 321 & 25.3 & $1.31(1.14-1.51)$ & 0.001 & $0.93(0.79-1.10)$ & 0.42 \\
\hline & Low eGFR without anemia & 108 & 31.7 & $1.80(1.42-2.28)$ & $<0.001$ & $0.97(0.73-1.29)$ & 0.85 \\
\hline & Anemia with low eGFR & 74 & 36.3 & $2.21(1.65-2.96)$ & $<0.001$ & $1.17(0.83-1.64)$ & 0.38 \\
\hline \multirow{4}{*}{ All-cause death } & None of both & 588 & 9.8 & 1 & - & 1 & - \\
\hline & Anemia with normal eGFR & 229 & 15.3 & $1.66(1.41-1.95)$ & $<0.001$ & $1.14(0.94-1.39)$ & 0.19 \\
\hline & Low eGFR without anemia & 151 & 30.7 & $4.07(3.30-5.02)$ & $<0.001$ & $1.50(1.14-1.97)$ & 0.004 \\
\hline & Anemia with low eGFR & 113 & 35.7 & $5.09(3.99-6.51)$ & $<0.001$ & $2.64(1.94-3.59)$ & $<0.001$ \\
\hline \multirow{4}{*}{ Any stroke reoccurrence } & None of both & 876 & 15.3 & 1 & - & 1 & - \\
\hline & Anemia with normal eGFR & 288 & 20.3 & $1.42(1.22-1.64)$ & $<0.001$ & $1.10(0.94-1.29)$ & 0.23 \\
\hline & Low eGFR without anemia & 121 & 28.1 & $2.18(1.74-2.72)$ & $<0.001$ & $1.14(0.89-1.47)$ & 0.30 \\
\hline & Anemia with low eGFR & 91 & 32.0 & $2.62(2.02-3.40)$ & $<0.001$ & $1.42(1.06-1.91)$ & 0.017 \\
\hline
\end{tabular}

*Adjusted for age; sex; medical history of hypertension, diabetes, hyperlipidemia, current smoking, heavy drinking, stroke history, coronary arterial disease, heart failure, atrial fibrillation, family stroke history; baseline NIHSS score; stroke etiology; fasting glucose; in-hospital use of antiplatelets, statin, hypoglycemic agents or hypertensive agents; in-hospital pneumonia and urinary tract infection.

†Poor functional outcome denotes modified Rankin scale score 3-6.

‡Dependence, modified Rankin scale score 3-5

Conclusion: Ischemic stroke patients with concurrent low eGFR and anemia had increased risks of poor functional outcome, mortality and stroke reoccurrence, but not dependence during 1-year follow up. 